\title{
A LINEAR INITIAL-VALUE PROBLEM
}

\section{J. S. MAC NERNEY}

The problem before us today is connected with the role of the Stieltjes integral concept as related to linear differential systems. For example, suppose that $P$ is a numerical function defined on the real line and Lebesgue integrable on each interval, and each of $c$ and $Y$ is a real number. It is commonplace that the function $F$, absolutely continuous on each interval, described by the differential requirement

$$
F(c)=Y \text { and } F^{\prime}=F \cdot P \text { almost everywhere, }
$$

is equivalently described by integrating both sides of the differential equation from the initial point $c$ (the integration being in the sense of Lebesgue); another description, however, is provided by the ordinary Stieltjes integral requirement

$$
F(z)=Y+\int_{c}^{z} F \cdot d \phi \text { for each real number } z,
$$

where $\phi$ is any function, absolutely continuous on each interval, having $P$ as its almost everywhere derivative. Similar translation is feasible, of course, with finite systems of first order equations: $Y$ and the functions $F$ and $P$ and $\phi$ are taken to be matrix valued, and juxtaposition is then interpreted as matrix multiplication.

In connection with such systems of first order linear equations, there are "interface problems" wherein prescribed discontinuities are imposed on the otherwise locally absolutely continuous function $F$ in equation (1) (the 1955 work of F. W. Stallard [14] and of T. J. Pignani and W. M. Whyburn [11] is basic in that area). Some types of interface singularities can also be introduced in these systems via the function $\phi$ in equation (2); recently Stallard [15] has succeeded in translating some of these modified versions of (2) back into the differential equation setting. I regret that his important work does not fall within the scope of my subsequent remarks here today.

I wish to focus attention on possible modifications of the integral in system (2) which may arise when the continuity condition on $\phi$ is dropped (a bounded variation condition being retained), and on related modifications in the "adjoint system," which, in the continuous case, inherits the form,

An address delivered before the Tallahassee meeting of the Society on November 16, 1962, by invitation of the Committee to Select Hour Speakers for Southeastern Sectional Meetings; received by the editors December 30, 1962. 


$$
G(x)=Y+\int_{x}^{0} d \phi \cdot G \text { for each real number } x,
$$

from the classical differential equation counterpart. We shall see that there is an axiomatic description of these conjoined systems; that there may be different modifications of the integral in (2) and that in (3), consistent with the existence of a function $W$ which is defined on the plane and provides unique solutions in the form

$$
F(z)=Y \cdot W(c, z) \text { and } G(x)=W(x, c) \cdot Y ;
$$

and that there is a somewhat larger problem suggested by this analysis.

Some of the results which I shall present here today are new, and new theorems will be designated by Arabic numerals. Theorems which are paraphrases of those already in the literature are to be designated by letters from the Roman alphabet, and the principal references for these are: the 1954 paper by H.S. Wall [17] in Archiv der Mathematik (also, see [16]), the 1955 paper of mine [4] in Annals of Mathematics, and a current paper of mine [8] in the Illinois Journal of Mathematics. I call attention, also, to the closely related 1959 paper by T. H. Hildebrandt [2] in the latter journal, and to the 1955 paper of mine [5] in the Journal of the Elisha Mitchell Scientific Society.

1. A prototype of our investigation here is contained in $\mathrm{H}$. S. Wall's theory of harmonic matrices. Let $I$ denote the identity function on the real line $S, n$ be a positive integer, and the numerals 0 and 1 denote, respectively, the zero and identity matrices of order $n$. Let $\Phi_{n}$ denote the class of all $n$-by- $n$ matrices $\phi$ of complex functions defined on $S$, continuous and of bounded variation on each interval, such that $\phi(0)=0$. Let $H_{n}$ denote the class of all $n$-by- $n$ matrices $W$ such that each $W_{p q}$ is a continuous complex function defined on $S \times S$ with, for each number $y, W_{p q}(I, y)$ of bounded variation on each inter$\mathrm{val}$, and such that if each of $x, y$, and $z$ is a real number then

$$
W(x, y) \cdot W(y, z)=W(x, z) \text { and } W(x, x)=1 ;
$$

this is the class of $n$-by- $n$ harmonic matrices, and the basic theorem concerns a one-to-one correspondence between $\Phi_{n}$ and $H_{n}$.

Theorem A. [17, Theorem 1 and Corollary 1.3.] There is a reversible function $\varepsilon_{0}$ from $\Phi_{n}$ onto $H_{n}$ which is established by the condition that

$$
W(x, z)=1+\int_{x}^{z} d \phi \cdot W(I, z)
$$


for all $\{x, z\}$ in $S \times S$; moreover, for each $c$ in $S$ and each $n$-by- $n$ complex matrix $Y$, if $W=\varepsilon_{0}(\phi)$ then the formulas (4) describe the only $n$-by-n matrices $F$ and $G$ of continuous complex functions defined on $S$ and satisfying (2) and (3), respectively.

In this theorem, the $W$ is obtained from the $\phi$ by successive substitutions in (6) $[17$, p. 161] generating a convergent Peano-series expansion (also, see $[8, \S 6]$ ) from which one can see the exponential nature of $W$ in case the matrix values of $\phi$ permute multiplicatively. There is another theorem, however, which provides explicit formulas for the function $\varepsilon_{0}$. Here, and hereafter, $d \phi(x, z)$ means $\phi(z)-\phi(x)$.

Theorem B. If $W=\varepsilon_{0}(\phi)$ then, for each $\{x, z\}$ in $S \times S$,

(i) $\left[17\right.$, Corollary 1.1] if c is a number, $d \phi(x, z)=\int_{x}^{z} W(I, c) \cdot d W(c, I)$.

(ii) $\left[4\right.$, Theorem 3.5] $W(x, z)={ }_{x} \Pi^{z}[1+d \phi]$, the limit (with decreasing norm of subdivisions) of continued matrix products of the form,

$$
\left[1+d \phi\left(s_{0}, s_{1}\right)\right]\left[1+d \phi\left(s_{1}, s_{2}\right)\right] \cdots\left[1+d \phi\left(s_{n-1}, s_{n}\right)\right],
$$

for monotone sequences $\left\{s_{p}\right\}_{0}^{n}$ with $s_{0}=x$ and $s_{n}=z$.

Now, let us consider introducing a norm on our algebra of $n$-by- $n$ complex matrices, such as (for example)

$$
|Y|=\max _{p} \sum_{q}\left|Y_{p q}\right| ;
$$

various relationships would hold, as here indicated:

$$
\left\{\begin{array}{l}
|Y|>0 \text { if } Y \neq 0, \quad|0|=0, \quad|1|=1, \\
|X+Y| \leqq|X|+|Y| \text { and }|X Y| \leqq|X||Y|, \\
\text { and }|c X|=|c||X| \text { for each number } c,
\end{array}\right.
$$

and the notions of continuity, convergence, and bounded variation could be equivalently discussed in terms of this norm. I prefer, and intend, to assume merely that we have a real normed algebra $N$, with additive and multiplicative identity elements denoted by 0 and 1 , that the relationships (8) hold for our norm on $N$, and that $N$ is complete with respect to this norm (so that we have what is sometimes called a real Banach algebra).

We do not insist on the finite dimensionality of our matrix algebra, and it is patently true that Theorems $\mathrm{A}$ and $\mathrm{B}$ have natural extensions to this setting [17, Theorem $4 ; 4$, Theorems $3.1,3.2,3.5]$.

2. Considering, for a moment, the problem of modifying the Stieltjes integral as indicated earlier, let us agree that all integrals henceforth will be determined as limits in the sense of successive refinements of 
subdivisions (styled the sigma-limit by Hildebrandt [2] and others), instead of requiring limits with decreasing norm of subdivisions. For functions $F$ and $\phi$ from the real line $S$ to the complete normed algebra $N$, of bounded variation on each interval, the possibilities include (and the existence is easily checked in each case) the Cauchy left and right integrals $[8, \S 4]$ and the interior integral considered by $S$. Pollard ("restricted" integral, [12, p. 123]):
(L) $\int_{x}^{z} F \cdot d \phi \sim F(x) d \phi(x, z)$,
(R) $\int_{x}^{z} F \cdot d \phi \sim F(z) d \phi(x, z)$,
(I) $\int_{x}^{z} F \cdot d \phi \sim F(s) d \phi(x, z)$
$(x<s<z$ or $x>s>z)$,

with each $\int_{x}^{x}=0$. Here, I have indicated, in each case, the one-term approximating sum, considering it inappropriate to belabor you with the formal definitions which can be readily inferred. Each integral generates a function $V$ on $S \times S$ with the property that if $x \leqq y \leqq z$ then $V(x, y)+V(y, z)=V(x, z)$, but only in the third case does $V$ share with $d \phi$ the property that $V(x, y)=-V(y, x)$.

I say that functions $V$ and $W$ (from $S \times S$ to any algebraic ring) are order-additive and order-multiplicative, respectively, $[8, \S 1]$ provided that if either $x \leqq y \leqq z$ or $x \geqq y \geqq z$ then

$$
V(x, y)+V(y, z)=V(x, z) \text { and } W(x, y) W(y, z)=W(x, z) .
$$

Let $\mathcal{O} Q^{+}$denote the class of all order-additive functions from $S \times S$ to the set of nonnegative real numbers, and $0 \mathrm{rT}^{+}$denote the class of all order-multiplicative functions from $S \times S$ to the set of real numbers not less than 1.

There is an obvious one-to-one correspondence between $\theta a^{+}$and $\Theta \mathfrak{N C}^{+}$, as determined by the exponential function: for $\alpha$ in $\Theta Q^{+}$,

$$
\mu(x, z)=\operatorname{Exp}\{\alpha(x, z)\} \quad \text { for all }\{x, z\} \text { in } S \times S ;
$$

this, however, seems not to be the "right" one for our purposes.

Theorem C. [8, Theorem 2.2.] There is a reversible function $\varepsilon^{+}$ from $\mathcal{O} Q^{+}$onto $\mathcal{O N} C^{+}$to which $\{\alpha, \mu\}$ belongs only in case one of the following holds:

(i) $\mu$ is in $0 \mathrm{~T}^{+}$and $\alpha$ is defined on $S \times S$ by

$$
\alpha(x, z)=\text { g.l.b. } \sum_{1}^{n}\left[\mu\left(s_{p-1}, s_{p}\right)-1\right]
$$

for all monotone sequences $\left\{s_{p}\right\}_{0}^{n}$ with $s_{0}=x$ and $s_{n}=z$; 
(ii) $\alpha$ is in $\theta a^{+}$and $\mu$ is defined on $S \times S$ by

$$
\mu(x, z)=\text { l.u.b. } \prod_{1}^{n}\left[1+\alpha\left(s_{p-1}, s_{p}\right)\right]
$$

for all monotone sequences $\left\{s_{p}\right\}_{0}^{n}$ with $s_{0}=x$ and $s_{n}=z$.

This is our first analogue of Theorem B for a case in which discontinuities are allowed. Since the indicated sums and products are, respectively, nonincreasing and nondecreasing with successive refinements of subdivisions, I rewrite the formulas from Theorem $\mathrm{C}$ as

$$
\alpha(x, z)={ }_{x} \sum^{z}[\mu-1] \text { and } \mu(x, z)={ }_{x} \Pi^{z}[1+\alpha],
$$

the continuously continued sum and the continuously continued product being limits, in the sense of successive refinements of subdivisions, of the indicated continued sums and products. From this you will infer, immediately and correctly, the meanings of

$$
{ }_{x} \sum^{z}[W-1] \text { and }{ }_{x} \Pi^{z}[1+V]
$$

for functions $V$ and $W$ from $S \times S$ to our algebra $N$ (also, see $[8, \S 1]$ ). In the product, of course, the multiplication should be "from left to right" as in (ii) of Theorem B.

3. For our next analogue of Theorem B, I must mention two special classes of order-additive functions and of order-multiplicative functions, respectively, from $S \times S$ to $N$. Let $O Q$ denote the class of all order-additive functions $V$ from $S \times S$ to $N$ such that there is a member $\alpha$ of $\theta a^{+}$with the property that $|V| \leqq \alpha$, that is,

$$
|V(x, z)| \leqq \alpha(x, z) \quad \text { for all }\{x, z\} \text { in } S \times S .
$$

Let $O$ Ir denote the class of all order-multiplicative functions $W$ from $S \times S$ to $N$ such that there is a member $\mu$ of $0 \pi^{+}$with the property that $|W-1| \leqq \mu-1$, that is,

$$
|W(x, z)-1| \leqq \mu(x, z)-1 \quad \text { for all }\{x, z\} \text { in } S \times S .
$$

One should notice that, for each $x$ in $S$, if $W$ is in 0 Ir then $W(x, x)=1$ in $N$, since if $\mu$ is in $0 T^{+}$then $\mu(x, x)^{2}=\mu(x, x) \geqq 1$. Our second analogue of Theorem B is this:

THEOREM D. [8, Theorem 3.3.] There is a reversible function $\&$ from $O Q$ onto OTh such that each of the following is a necessary and sufficient

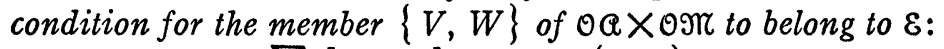

(i) $V(x, z)={ }_{x} \sum_{z}[W-1]$ for each $\{x, z\}$ in $S \times S$.

(ii) $W(x, z)={ }_{x} \Pi^{z}[1+V]$ for each $\{x, z\}$ in $S \times S$. 
(iii) There is an ordered pair $\{\alpha, \mu\}$ in $\varepsilon^{+}$such that, for $\{x, z\}$ in $S \times S$,

$$
|W(x, z)-1-V(x, z)| \leqq \mu(x, z)-1-\alpha(x, z) .
$$

The existence of the continuously continued sum and product which appear in Theorem $\mathrm{D}$ may be deduced from the completeness of our normed algebra $N$, by establishing inequalities of the form

$$
\begin{aligned}
& \left|\sum_{t}[W-1]-\sum_{t}[W-1]\right| \leqq \sum_{t}[\mu-1]-\sum_{t}[\mu-1] \text { and } \\
& \left|\prod_{s}[1+V]-\prod_{t}[1+V]\right| \leqq \prod_{t}[1+\alpha]-\prod_{t}[1+\alpha],
\end{aligned}
$$

where $s$ is an ordered subdivision of $\{x, z\}$ and $t$ is a refinement of $s$, $\sum_{s}$ denotes an approximating sum over $s$, etc. The inequalities (14) are based on the observation that

$$
\left|\prod_{1}^{n}\left[1+B_{p}\right]-\left(1+\sum_{1}^{n} B_{p}\right)\right| \leqq \prod_{1}^{n}\left[1+b_{p}\right]-\left(1+\sum_{1}^{n} b_{p}\right)
$$

provided that $\left|B_{p}\right| \leqq b_{p}(p=1, \cdots, n)$ [8, proof of Theorem 3.1], an inequality which seems to have escaped the attention of earlier workers in the field of multiplicative integration (for instance, see the 1947 paper by P. R. Masani [9] in the Transactions of the Society).

To see the sense in which this last theorem actually includes Theorem B, let us note that for the function $\phi$ from $S$ to $N$ to be of bounded variation on each interval it is necessary and sufficient that $d \phi$ should belong to the class $\mathcal{O Q}$; indeed, for such a $\phi$, we can define the dominating $\alpha$ in $\theta Q^{+}$to have for its value, at each $\{x, z\}$ in $S \times S$, the total variation of $\phi$ from $x$ to $z$. My use of the nonnegative dominating functions from $\mathcal{O Q}^{+}$and $\mathcal{O N}{ }^{+}$, in the context of Theorem $\mathrm{D}$, is closely related to J. W. Neuberger's use of variation functions and closing functions in his 1958 paper [10], in the Pacific Journal of Mathematics, dealing with nonlinear versions of the problems we are considering here today.

4. Now, we come to our first (possibly "discontinuous") analogue of H. S. Wall's Theorem A. Let $O Q$ denote the class of all functions $F$ from $S$ to $N$ such that $d F$ belongs to the class $O Q$ : as I have just noted, this is precisely the class of functions from $S$ to $N$ which are of bounded variation on each interval, corresponding in a natural way to the fully additive members of $\theta a$. The integrals we use for 
modified versions of our original equations (2) and (3) are the left and right integrals, respectively, with "approximate definition"

$$
(L) \int_{x}^{z} F \cdot V \sim F(x) V(x, z) \text { and }(R) \int_{x}^{x} V \cdot G \sim V(x, z) G(z)
$$

(in the sense previously explained); these are readily shown to exist for $V$ in $\mathcal{O Q}, F$ and $G$ in $\mathcal{O B}$, and $\{x, z\}$ in $S \times S$ [8, Lemmas 4.1 and 4.3]. The theorem we now have is this:

THEOREM E [8, Theorems 4.1 and 4.2.] If $V$ is in $\mathcal{O} a$ and $W=\varepsilon(V)$ then, for each $c$ in $S$ and $Y$ in $N$, both the following hold:

(i) The only member $F$ of $\Theta B$ such that $F(z)=Y+(L) \int_{c}^{z} F \cdot V$, for each $z$ in $S$, is the $F$ defined by $F(z)=Y \cdot W(c, z)$.

(ii) The only member $G$ of $O B$ such that $G(x)=Y+(R) \int_{x}^{c} V \cdot G$, for each $x$ in $S$, is the $G$ defined by $G(x)=W(x, c) \cdot Y$.

These Theorems C, D, and E have been established in the somewhat broader setting in which the real line $S$ is replaced by any linearly ordered set [8], a result which may be of some interest to those concerned with the computational aspects of this analysis. An example, of the use to which we can put the richer topological structure of the real line, is afforded by the following observation. If $\beta$ is a member of $\mathcal{O} Q^{+}$and $\{x, z\}$ is in $S \times S$ with $x<z$ then, for each positive number $b$, there is an increasing sequence $\left\{t_{p}\right\}_{0}^{n}$ with $t_{0}=x$ and $t_{n}=z$ such that

$$
\beta\left(t_{p-1}+, t_{p}-\right)<b \quad \text { for } p=1, \cdots, n,
$$

the left member of this inequality being the $\beta$-measure of the open segment $\left(t_{p-1}, t_{p}\right)$. This inequality follows from the fact that a nondecreasing real function on a number-interval is the uniform limit of a sequence of simple step functions (a fact which, incidentally, characterizes the completeness of the real line).

By using this observation, the existence and uniqueness results of T. H. Hildebrandt [2], to which earlier allusion was made, can be reduced to the left-right integral context of Theorem E $[8, \S 10]$. The Stieltjes integral modification so effectively used by Hildebrandt is a version of the Lebesgue-Stieltjes integral studied by W. H. Young [18]: for $F$ in $O Q$ and $V$ in $O Q$, an "approximate definition" in the present setting is

$$
\text { (Y) } \int_{x}^{z} F \cdot V \sim\left\{\begin{array}{l}
F(x) V(x, x+)+F(y) V(x+, z-)+F(z) V(z-, z) \text { or } \\
F(x) V(x, x-)+F(y) V(x-, z+)+F(z) V(z+, z),
\end{array}\right.
$$

according as $x<y<z$ or $x>y>z$, and the obvious analogue for $(Y) \int_{x}^{2} V \cdot G$, with $(Y) \int_{x}^{x}=0$. 
What I intend, now, is to give an axiomatic description of some of the systems for which this same reduction process (to the context of Theorem E) can be carried out.

5. The axiomatic description is fourfold, the first part being the Linearity Axiom:

Axıom I. Each of $K_{1}$ and $K_{2}$ is an additive function from $O B$ into $\mathcal{O Q}$ and, for each $U$ in $\Theta B$ and each $\{X, Z\}$ in $N \times N$,

$$
K_{1}[X U]=X K_{1}[U] \text { and } K_{2}[U Z]=K_{2}[U] Z .
$$

Clearly, $O Q$ and $O B$ are additive families of functions and this axiom is satisfied by all appropriate pairs of integrals already mentioned, as is also the Boundedness Axiom:

AxIom II. There is a member $\lambda$ of $\mathcal{O Q}^{+}$with the property that, if $U$ is in $\Theta \circ$ and $\{x, z\}$ is in $S \times S$ and $m$ is a number such that $|U(y)|$ $\leqq m$ for all $y$ in $S$ such that $x \leqq y \leqq z$ or $x \geqq y \geqq z$, then

$$
\left|K_{1}[U](x, z)\right| \leqq m \lambda(x, z) \text { and }\left|K_{2}[U](x, z)\right| \leqq m \lambda(x, z) \text {. }
$$

Looking ahead, I suggest that we are to consider the possible existence, for each $c$ in $S$, of functions $F$ and $G$ belonging to the class $O_{B}$ such that

$$
F(z)=F(c)+K_{1}[F](c, z) \text { and } G(x)=G(c)+K_{2}[G](x, c)
$$

for all $x$ and $z$ in $S$, and of a function $W$ belonging to the class 0 Tr and providing the $F$ and $G$ for (19) in the now familiar form

$$
F(z)=F(c) W(c, z) \text { and } G(x)=W(x, c) G(c) .
$$

In investigating the nature of $K_{1}$ and $K_{2}$, the following notational device is useful: in any appropriate context, the numeral 1 also denotes the constant function from $S$ to $N$ having only the value 1 , $1_{x}$ (for $x$ in $S$ ) denotes the function from $S$ to $N$ having the value 1 at $x$ and the value 0 elsewhere, and $0_{x}$ denotes the function $1-1_{x}$ having the value 0 at $x$ and the value 1 elsewhere on $S$.

Now, a moment's reflection shows that, for example, if $x<z$ then

$$
K_{1}\left[1_{x}\right](x, z)=K_{1}\left[1_{x}\right](x, x+) \text { and } K_{1}\left[1_{z}\right](x, z)=K_{1}\left[1_{z}\right](z-, z) \text {, }
$$

with similar relations for $x>z$ and for $K_{2}$ in place of $K_{1}$. Also, if $U$ is in $O Q B$ and $\beta$ is a member of $O Q^{+}$such that $|d U| \leqq \beta$ then, for each triple $\{x, y, z\}$ of numbers with $y$ between $x$ and $z$, the difference $U(x) K_{1}\left[1_{x}\right](x, z)+U(y) K_{1}\left[0_{x}-1_{z}\right](x, z)+U(z) K_{1}\left[1_{z}\right](x, z)-K_{1}[U](x, z)$ has norm not exceeding $\beta(x+, z-) \lambda(x, z)$ or $\beta(x-, z+) \lambda(x, z)$, according as $x<z$ or $x>z$. These considerations lead directly to a representation for $K_{1}$ and $K_{2}$. 
TheOREM 1. With $V=K_{1}[1]$ and $\vec{V}=K_{2}[1]$, there exists a member $\{A, B\}$ of $0 Q \times 0 Q$ and a member $\{\bar{A}, \bar{B}\}$ of $0 a \times 0 Q$ such that if $U$ is in $O B$ and $\{x, z\}$ is in $S \times S$ then these formulas hold:

$$
\begin{aligned}
& K_{1}[U](x, z) \\
& =(L) \int_{x}^{x} U \cdot A+(I) \int_{x}^{z} U \cdot(-A+V-B)+(R) \int_{x}^{x} U \cdot B \text { and } \\
& K_{2}[U](x, z) \\
& =(L) \int_{x}^{z} \bar{A} \cdot U+(I) \int_{x}^{x}(-A+\bar{V}-\bar{B}) \cdot U+(R) \int_{x}^{x} \bar{B} \cdot U .
\end{aligned}
$$

In indication of proof, beyond the remarks preceding Theorem 1, I shall let it suffice to give a formula for one such ordered pair $\{A, B\}$ as is mentioned in the theorem. For each $x$ in $S$ let each of $A(x, x)$ and $B(x, x)$ be zero and, for each $z$ in $S$ different from $x$, let

$$
\begin{array}{llll}
A(x, z) & =\sum_{x \leq y<z} K_{1}\left[1_{y}\right](y, y+) & \text { or } & \sum_{x \geq y>z} K_{1}\left[1_{y}\right](y, y-), \\
B(x, z)=\sum_{x<y \leq z} K_{1}\left[1_{y}\right](y-, y) & \text { or } & \sum_{x>y \geq z} K_{1}\left[1_{y}\right](y+, y)
\end{array}
$$

according as $x<z$ or $x>z$; the nature of the convergence is apparent from the observation that the sum of the norms of these elements of $N$ over any finite set of the $y$ 's is bounded by $\lambda(x, z)$.

I wish to make one more observation about the nature of Theorem 1: there is the obvious converse theorem to the effect that, for any $A$, $\bar{A}, B, \bar{B}, V$, and $\bar{V}$ in the class $\vartheta Q$, the formulas (23) define functions $K_{1}$ and $K_{2}$ which satisfy Axioms I and II.

6. As a preamble to the next axiom, I now offer a short computational procedure, coupled with some heuristic remarks. Supposing that $c$ is in $S$ and $F$ is a member of $O B$ such that

$$
F(z)=F(c)+K_{1}[F](c, z) \quad \text { for each } z \text { in } S,
$$

we find that, for $c \leqq x<z$,

$$
\begin{aligned}
& F(x+)-F(x)=F(x) K_{1}\left[1_{x}\right](x, x+)+F(x+) K_{1}\left[0_{x}\right](x, x+), \\
& F(x+)\left\{1-K_{1}\left[0_{x}\right](x, x+)\right\}=F(x)\left\{1+K_{1}\left[1_{x}\right](x, x+)\right\}, \\
& F(z)-F(z-)=F(z-) K_{1}\left[0_{z}\right](z-, z)+F(z) K_{1}\left[1_{z}\right](z-, z), \text { and } \\
& F(z)\left\{1-K_{1}\left[1_{z}\right](z-, z)\right\}=F(z-)\left\{1+K_{1}\left[0_{z}\right](z-, z)\right\}
\end{aligned}
$$

to make our initial-value problem (25) well posed, in the sense of arranging that the history of $F$ from $c$ through $x$ should determine its value "immediately thereafter" and that the history of $F$ prior to $z$ 
should determine its value at $z$, it seems reasonable to require that certain elements of $N$, appearing as factors of $F(x+)$ and $F(z)$ in the second and fourth lines of (26), should have multiplicative inverses in the algebra $N$; moreover, there should be two other such elements which arise for $c \geqq x>z$, and four involving $K_{2}$. This is the intent of the following Regularity Axiom:

Axıom III. For each $y$ in $S$, each of the following has a multiplicative inverse in the algebra $N$ :

$$
\begin{aligned}
& 1-K_{1}\left[0_{y}\right](y, y+) \text { and } 1-K_{1}\left[1_{y}\right](y-, y) \text {, } \\
& 1-K_{1}\left[0_{y}\right](y, y-) \text { and } 1-K_{1}\left[1_{y}\right](y+, y) \text {, } \\
& 1-K_{2}\left[1_{y}\right](y, y+) \text { and } 1-K_{2}\left[0_{y}\right](y-, y) \text {, and } \\
& 1-K_{2}\left[1_{y}\right](y, y-) \text { and } 1-K_{2}\left[0_{y}\right](y+, y) \text {. }
\end{aligned}
$$

I hasten to point out that this axiom is a restrictive imposition at not more than finitely many $y$ 's in any interval, because of the familiar fact that if $Z$ is in $N$ and $|Z|<1$ then $1-Z$ already has a multiplicative inverse in $N$. We now have enough axioms to effect a reduction to the context of Theorem $\mathrm{E}$, as follows.

THEOREM 2. There exists an ordered pair $\left\{V_{1}, V_{2}\right\}$ in $0 a \times 0 a$ such that, for each $c$ in $S$, if $F$ is in $O B$ then these are equivalent:

(i) $F(z)=F(c)+K_{1}[F](c, z)$ for each $z$ in $S$, and

(ii) $F(z)=F(c)+(L) \int_{c}^{2} F \cdot V_{1}$ for each $z$ in $S$;

whereas, if $G$ is in $O B$ then these are also equivalent:

(iii) $G(x)=G(c)+K_{2}[G](x, c)$ for each $x$ in $S$, and

(iv) $G(x)=G(c)+(R) \int_{x}^{c} V_{2} \cdot G$ for each $x$ in $S$.

I have shown elsewhere [8, Theorem 10.1] how to establish this result for the special case investigated by Hildebrandt [2], involving the integrals of $\mathrm{W}$. H. Young mentioned earlier in this lecture:

$$
K_{1}[F](c, z)=(Y) \int_{c}^{*} F \cdot d \phi \text { and } K_{2}[G](x, c)=(Y) \int_{x}^{0} d \phi \cdot G .
$$

That pattern of argument can be extended directly to our present setting, once the appropriate formulas for $V_{1}$ and $V_{2}$ are available. Omitting other details, I should like now to display these formulas, since they involve explicitly the inverses postulated in Axiom III. Letting $V=K_{1}[1]$ and $\bar{V}=K_{2}[1]$ as in Theorem 1, I define

$$
\begin{aligned}
V_{1}(x, z)= & V(x, z)+\sum_{x \leq y<z} V(y, y+)\left\{1-K_{1}\left[0_{y}\right](y, y+)\right\}^{-1} K_{1}\left[0_{y}\right](y, y+) \\
& +\sum_{x<y \leq z} V(y-, y)\left\{1-K_{1}\left[1_{y}\right](y-, y)\right\}^{-1} K_{1}\left[1_{y}\right](y-, y)
\end{aligned}
$$


or

$$
\begin{aligned}
= & V(x, z)+\sum_{x \geq y>z} V(y, y-)\left\{1-K_{1}\left[0_{y}\right](y, y-)\right\}^{-1} K_{1}\left[0_{y}\right](y, y-) \\
& +\sum_{x>y \geq z} V(y+, y)\left\{1-K_{1}\left[1_{y}\right](y+, y)\right\}^{-1} K_{1}\left[1_{y}\right](y+, y),
\end{aligned}
$$

according as $x<z$ or $x>z$, and similarly

$$
\begin{aligned}
V_{2}(x, z)= & \bar{V}(x, z)+\sum_{x \leqq y<z} K_{2}\left[1_{y}\right](y, y+)\left\{1-K_{2}\left[1_{y}\right](y, y+)\right\}^{-1} \bar{V}(y, y+) \\
& +\sum_{x<y \leqq z} K_{2}\left[0_{y}\right](y-, y)\left\{1-K_{2}\left[0_{y}\right](y-, y)\right\}^{-1} \bar{V}(y-, y)
\end{aligned}
$$

or

$$
\begin{aligned}
= & \bar{V}(x, z)+\sum_{x \geqq y>z} K_{2}\left[1_{y}\right](y, y-)\left\{1-K_{2}\left[1_{y}\right](y, y-)\right\}^{-1} \bar{V}(y, y-) \\
& +\sum_{x>y \geqq z} K_{2}\left[0_{y}\right](y+, y)\left\{1-K_{2}\left[0_{y}\right](y+, y)\right\}^{-1} \bar{V}(y+, y),
\end{aligned}
$$

with $V_{1}(x, x)=V_{2}(x, x)=0$. The convergence is of the same nature as that indicated for the pair $\{A, B\}$ in (24) in connection with Theorem 1 .

7. We are now almost ready to join together the integral operations $K_{1}$ and $K_{2}$. Let us first observe a corollary result, to Theorem 2, which ties our investigation here back to the continuous case of Wall's harmonic matrices and to Hildebrandt's investigation of the Young integral, and to my own 1955 investigation [5] involving the Stieltjes mean integrals $[13 ; 8, \S 7]$ in this context:

$$
\text { (M) } \int_{x}^{z} F \cdot d \phi \sim \frac{F(x)+F(z)}{2}[\phi(z)-\phi(x)] .
$$

An argument can be based on the unique solvability provided by Theorems $E$ and 2, precisely as in the published proof of Wall's first theorem [17, pp. 161-162], to produce the following result.

Theorem 3. If $W_{1}=\varepsilon\left(V_{1}\right)$ and $W_{2}=\varepsilon\left(V_{2}\right)$ and, for each $U$ in $O B$, each of $K_{1}[U]$ and $K_{2}[U]$ is a member $C$ of $\mathcal{O}$ a such that

$$
C(x, y)+C(y, z)=C(x, z) \quad \text { for all } x, y \text {, and } z \text { in } S,
$$

then each of $W_{1}$ and $W_{2}$ is a member $W$ of orT such that

$$
W(x, y) \cdot W(y, z)=W(x, z) \quad \text { for all } x, y, \text { and } z \text { in } S .
$$

Under what circumstances, we may ask, does there exist a single function $W$ in the class OrT such that the solutions $F$ and $G$ of our $K_{1}-K_{2}$ initial-value problem are both given in terms of $W$, as in the earlier problems involving differential equations or harmonic matri- 
ces? By Theorem $\mathrm{E}$ and Theorem 2, the answer is, of course, that $V_{1}$ should be the same as $V_{2}$ : this condition is both necessary and sufficient. Let us make the simplifying assumption that $\bar{V}$ is $V$ in our formulas, that is, that $K_{2}$ [1] is $K_{1}[1]$.

Now, for $V_{1}$ to be the same as $V_{2}$, it is necessary and sufficient that for each $y$ in $S$, symbolically,

$$
\text { "V } V_{2}=V_{1} \text { at }(y-, y),(y, y+),(y+, y), \text { and }(y, y-), "
$$

and, for example, in the case of the first "pair" certain equivalent conditions are easily found to be

$$
\begin{aligned}
& \left\{1-K_{2}\left[0_{y}\right](y-, y)\right\}^{-1} V(y-, y)=V(y-, y)\left\{1-K_{1}\left[1_{y}\right](y-, y)\right\}^{-1} \text { or } \\
& V(y-, y) K_{1}\left[1_{y}\right](y-, y)+K_{2}\left[1_{y}\right](y-, y) V(y-, y)=V(y-, y)^{2} .
\end{aligned}
$$

Each of the other three cases can be reduced to this latter form, and so we are led to the Conjunction Axiom:

AxIom IV. $K_{1}[1]=K_{2}[1]=V$ and, for each $y$ in $S$,

$$
J_{y}(y-, y)=J_{y}(y, y+)=J_{y}(y+, y)=J_{y}(y, y-)=0,
$$

where $J_{y}=V \cdot K_{1}\left[1_{y}\right]+K_{2}\left[1_{y}\right] \cdot V-V^{2}$.

It is time to summarize some of the main results of our investigation up to this point.

TheOREM 4. Assuming Axioms I through IV, there exists a function $W$ in the class OIT with the property that, for each c in $S$, if each of $F$ and $G$ is a function from the real line $S$ to the algebra $N$ then

(i) $F$ belongs to $\Theta B$ and $F(z)=F(c)+K_{1}[F](c, z)$ for each $z$ in $S$ only in case $F(z)=F(c) W(c, z)$ for each $z$ in $S$, and

(ii) $G$ belongs to $O B$ and $G(x)=G(c)+K_{2}[G](x, c)$ for each $x$ in $S$ only in case $G(x)=W(x, c) G(c)$ for each $x$ in $S$.

8. It seems of interest now to enumerate some of the ordered pairs $\left\{K_{1}, K_{2}\right\}$ which satisfy all four of the axioms (Linearity, Boundedness, Regularity, and Conjunction). All such pairs, of course, must be available from among the representations given in Theorem 1 , with $\bar{V}$ the same as $V$.

It should come as no surprise that one pair is

$$
K_{1}[F](x, z)=(L) \int_{x}^{z} F \cdot V \text { and } K_{2}[G](x, z)=(R) \int_{x}^{z} V \cdot G \text {. }
$$

In this case, of the left and right integrals, no added conditions are imposed by Axiom III. I should mention that in Hildebrandt's investigation, using the Young integral in both places, it was found that in general $W_{1}$ is different from $W_{2}$, that is, our fourth axiom 
fails. It turns out, now, that the interior integral and the Young integral form a natural pair, and I list also the typical elements of $N$ required to have inverses:

$$
\begin{gathered}
K_{1}[F](x, z)=(I) \int_{x}^{z} F \cdot V \text { and } K_{2}[G](x, z)=(Y) \int_{x}^{z} V \cdot G \\
1-V(y, y+), \quad 1-V(y, y-) .
\end{gathered}
$$

In this case, the conditions contemplated in Theorem 3 hold provided $V$ has the form $d \phi$ for some function $\phi$ in $\mathcal{O}$ B.

This latter remark applies also to the next, and final, instance I shall cite here: an instance in which the same integration process can be used in both places. The instance is that of the mean integral (also called the Stieltjes mean sigma integral), and again I list the typical elements of $N$ required to have inverses:

$$
\begin{aligned}
& K_{1}[F](x, z)=(M) \int_{x}^{z} F \cdot V \text { and } K_{2}[G](x, z)=(M) \int_{x}^{z} V \cdot G \\
& 1-\frac{1}{2} V(y-, y), 1-\frac{1}{2} V(y, y+), 1-\frac{1}{2} V(y+, y), 1-\frac{1}{2} V(y, y-) .
\end{aligned}
$$

As well as having arisen in earlier investigations of mine $[5 ; 6 ; 7]$ concerning quasi-harmonic matrices, these mean integrals also arise in a natural way in connection with W. H. Ingram's notion of the "jump-differential" [3]; indeed, it may very well be that Ingram has already solved the initial-value problem for finite systems of jumpdifferential equations, using the type of hypothesis indicated here in (33).

9. I remark, in passing, that there is a technique $[8, \S 5]$ for treating nonhomogeneous analogues of systems such as we have considered here, for instance

$$
G(x)=G(c)+(R) \int_{x}^{c} V \cdot G+Q(x, c),
$$

where each of $V$ and $Q$ is a member of the class $O Q$; the idea is to express this as a homogeneous system in an algebra of 2-by-2 matrices with elements in $N$. In the presence of Axioms I through III, that same technique carries over to give corresponding existence and uniqueness theorems for such a system as

$$
G(x)=G(c)+K_{2}[G](x, c)+Q(x, c) ;
$$

the details of the analysis would carry us too far afield if discussed at this time.

The result, however, is germane to the question of what we have 
gained by allowing the algebra $N$ to be infinite dimensional, and it is to this question that I would like to address myself for a few minutes. One possible application, similarly noticed by T. H. Hildebrandt (oral communication, May 1960), is in the area of integral equations of what might be called Stieltjes-Volterra type; an instance is

$$
u(x)=(R) \int_{x}^{c} d H(x, I) \cdot u+h(x),
$$

where $H$ and $h$ are numerical functions defined, respectively, on $S \times S$ and on $S$, and where it is desired to determine a numerical function $u$. I have no theorems to present at this time, and will indicate only the formalities of the idea.

If we have a suitably convergent expansion for $H$, then we may rewrite (36) in the form

$$
\begin{aligned}
u(x) & =\sum A_{n}(x) g_{n}(x)+h(x), \text { where } \\
H(x, y) & =\sum A_{n}(x) B_{n}(y) \text { and } g_{n}(x)=(R) \int_{x}^{o} d B_{n} \cdot u .
\end{aligned}
$$

Operating on both sides of (37), we might then obtain an infinite system of linear equations

$$
\begin{aligned}
g_{m}(x) & =\sum_{n}(R) \int_{x}^{c} V_{m n} \cdot g_{n}+q_{m}(x, c), \text { where } \\
V_{m n}(x, y) & =(R) \int_{x}^{y} d B_{m} \cdot A_{n} \text { and } q_{m}(x, y)=(R) \int_{x}^{y} d B_{m} \cdot h,
\end{aligned}
$$

a system analogous to (34). If the infinite sequence $g$ were found satisfying (38), then it might be substituted back in (37) to give a numerical function $u$.

These are the formalities. What is needed here seems to be some sort of balance, between strength of an expansion theorem for the function $H$, and strength of a topology for some infinite matrix algebra in which $V$ has its values.

10. Let us recall that in the development of the left and right integral theory (Theorems $\mathrm{C}, \mathrm{D}$, and $\mathrm{E}$ ) we could equally well have assumed of $S$ only that it was a linearly ordered set [8]. On the other hand, except in discussions involving the mean integral, no use is made of the availability of real numerical multipliers in the algebra $N$ : all of the theorems presented here hold equally well if $N$ is assumed only to be a complete normed ring (as in [8]). These facts lead me now to suppose only that $S$ is a linearly ordered set, and to relinquish 
the hypothesis of a norm for the ring $N$, in order to formulate a problem which seems a natural outgrowth of the preceding development.

Problem. Given an Abelian group $\{G,+\}$, a topology $T$ for $G, a$ subring $N$ of the ring of endomorphisms of $\{G,+\}$, and an orderadditive function $V$ from $S \times S$ to $N$, to investigate the existence of

(1) a function $W$ from $S \times S$ to $N$ such that if $g$ is in $G$ then

$$
W(x, z) g=g+\left\{(L) \int_{x}^{z} W(x, I) \cdot V\right\} g \quad \text { for all }\{x, z\} \text { in } S \times S,
$$

and (2) for each $c$ in $S$, nontrivial functions $u$ from $S$ to $G$ such that

$$
u(x)=u(c)+(R) \int_{x}^{o} V \cdot u \quad \text { for all } x \text { in } S,
$$

where, in each case, the indicated integral exists as a limit with respect to the topology $T$.

I should like to illustrate, in part, the intent of this twofold problem by exhibiting a solvable case which seems not to fall within the scope of the complete-normed-ring treatment.

For $\{G,+\}$ I take a Hilbert space, with inner product function $Q$; for $T$, the metric topology generated by the norm corresponding to $Q$; for $N$, a (necessarily commutative) ring of linear transformations (from $G$ into $G$ ) Hermitian with respect to $Q$, which is closed in the "strong operator topology" and to which the identity transformation 1 belongs. Inequalities between members of $N$ refer to the usual partial ordering:

$$
H_{2} \gg H_{1} \text { means } Q\left(g, H_{2} g\right) \geqq Q\left(g, H_{1} g\right) \quad \text { for all } g \text { in } G \text {. }
$$

Now, $\mathrm{OQ}^{++}$denotes the class of all order-additive functions $V$ from $S \times S$ to $N$ such that $V \gg 0$, and $O \mathscr{T} \tau^{++}$denotes the class of all ordermultiplicative functions $W$ from $S \times S$ to $N$ such that $W \gg 1$. Note that if $x$ is in $S$ and $W$ belongs to $0 \mathbb{T}^{++}$then $W(x, x)=1$ since it is idempotent and, therefore, is a projection $P$ such that $P \gg 1$. Recalling that the product of two nonnegative members of $N$ is again nonnegative, it is easy to see that arguments $[8, \S 2]$ establishing Theorem C carry over, mutatis mutandis, to establish the following.

THEOREM 5. There is a reversible function $\mathcal{E}^{++}$from $\theta a^{++}$onto $0 \mathrm{Tr}++$ such that each of the following is a necessary and sufficient condition for the member $\{V, W\}$ of $\Theta Q^{++} \times O \mathrm{NT}^{++}$to belong to $\varepsilon^{++}$:

(i) $V(x, z) g=\left\{{ }_{x} \sum^{z}[W-1]\right\} g$ for each $\{x, z\}$ in $S \times S$ and $g$ in $G$.

(ii) $W(x, z) g=\left\{\prod^{z}[1+V]\right\} g$ for each $\{x, z\}$ in $S \times S$ and $g$ in $G$. 
Direct computation, by analogy with the published argument for Theorem $\mathrm{E}[8, \S 4]$ serves to establish the fact that if $W=\varepsilon^{++}(V)$ then $W$ is a solution of Problem (1) and, for each $c$ in $S$ and nonzero $g$ in $G$, the function $W(I, c) g$ is a solution $u$ of Problem (2) in this special setting. Theorem 5 and related ideas have been significantly extended by R. H. Cox [1], who is presenting some of his results at this meeting, and this seems an appropriate place for me to stop.

\section{BIBLIOGRAPHY}

1. R. H. Cox, Integral equations in a Hilbert space, Abstract 594-33, Notices Amer. Math. Soc. 9 (1962), 468.

2. T. H. Hildebrandt, On systems of linear differentio-Stieltjes-integral equations, Illinois J. Math. 3 (1959), 352-373.

3. W. H. Ingram, The j-differential and its integral, Proc. Edinburgh Math. Soc. (2) 12 (1960), 85-93; (2) 13 (1962), 85-86.

4. J.S. Mac Nerney, Stieltjes integrals in linear spaces, Ann. of Math. (2) 61 (1955), 354-367.

5. - Continuous products in linear spaces, J. Elisha Mitchell Sci. Soc. 71 (1955), 185-200.

6. - Determinants of harmonic matrices, Proc. Amer. Math. Soc. 7 (1956), 1044-1046.

7. - Concerning quasi-harmonic operators, J. Elisha Mitchell Sci. Soc. 73 (1957), 257-261.

8. - Integral equations and semigroups, Illinois J. Math. 7 (1963), 148-173.

9. P. R. Masani, Multiplicative Riemann integration in normed rings, Trans. Amer. Math. Soc. 61 (1947), 147-192.

10. J. W. Neuberger, Continuous products and nonlinear integral equations, Pacific J. Math. 8 (1958), 529-549.

11. T. J. Pignani and W. M. Whyburn, Differential systems with interface and general boundary conditions, J. Elisha Mitchell Sci. Soc. 72 (1956), 1-14.

12. S. Pollard, The Stieltjes integral and its generalizations, Quart. J. Pure and Appl. Math. 49 (1920), 73-138.

13. H. L. Smith, On the existence of the Stieltjes integral, Trans. Amer. Math. Soc. 27 (1925), 491-515.

14. F. W. Stallard, Differential systems with interface conditions, Oak Ridge National Laboratory Publication No. 1876 (Physics), April 28, 1955.

15. - Functions of bounded variation as solutions of differential systems, Proc. Amer. Math. Soc. 13 (1962), 366-373.

16. H. S. Wall, Concerning continuous continued fractions and certain systems of Stieltjes integral equations, Rend. Circ. Mat. Palermo (2) 2 (1953), 73-84.

17. - Concerning harmonic matrices, Arch. Math. 5 (1954), 160-167.

18. W. H. Young, On integration with respect to a function of bounded variation, Proc. London Math. Soc. (2) 13 (1914), 109-150.

University of North Carolina at Chapel Hill 